\title{
AN IMPROVED ANALYSIS FOR THE HARMONIC DISTORTION OF MOS VOLTAGE-CONTROLLED-RESISTORS
}

\author{
MUHAMMAD TAHER ABUELMA'ATTI \\ King Fahd University of Petroleum and Minerals Box 203 Dhahran 31261 Saudi Arabia \\ (Received January 30, 1996; in final form March 29, 1996)
}

In this paper, a fourth-order polynomial expression is obtained for the nonlinear current-voltage characteristic of a MOS transistor operating in the triode region. Using this expression, closed-form expressions are obtained for the second-, third- and fourth-harmonic distortion of a MOS voltagecontrolled-resistors. The analytical expressions obtained in this paper can be used for a quantitative study of the effect of different parameters of the performance of MOS voltage-controlled-resistors.

\section{INTRODUCTION}

Voltage-controlled-resistors employing MOS transistors operating in their linear region are essential elements in realizing continuous time filters [1-3]. These resistors, however, exhibit nonlinearities that give rise to harmonic distortion. A circuit designer must, therefore, be able to accurately predict the harmonic performance of these resistors. An essential prerequisite for reliable prediction of the harmonic distortion is the accurate modelling of the MOS current-voltage characteristics in the linear region. While the model proposed by Merckel et al. [4] enjoys high accuracy, it is too complicated and cannot yield closed-form expressions for the harmonic distortion. A simplified version of this model was used in a modified version of SPICE to predict the harmonic performance of MOS voltagecontrolled resistor circuits [5]. No attempt has been reported, however, to use this simplified model to obtain closed-form expressions for the harmonic distortion. Although, the model proposed by White et al. [6] is simpler, and was successfully used to investigate the effectiveness of a range of linearization strategies of MOS resistors [7], it cannot yield closed-form expressions for the harmonic distortion. The simplified model proposed by Shoucair et al. [8] was successfully used to obtain closed-form expressions for the harmonic distortion of body driven MOSFET circuits $[8,9]$. At relatively low gate voltages, however, the accuracy of the model deteriorates [8].

In this paper, it will be shown that the simplified model proposed by Ryan [5] can be used to obtain closed-form expressions for the harmonic performance of MOS voltage-controlled resistors operating in the linear region. These expressions are valid over a wide range of gate-source and drain-source voltages. 


\section{ANALYSIS}

Assuming that the mobility degradation due to the lateral field in the MOS channel is zero, the current-voltage characteristic of the MOS transistor can be expressed by [5]

$I_{D S}=\beta \int_{V_{S}}^{V_{D}}\left(\frac{V_{G}-V_{F B}-\phi_{B}-V-\gamma\left(V+V_{B}+\phi_{B}\right)^{1 / 2}}{1+\theta\left(V_{G}-V_{F B}-\phi_{B}-V+\gamma\left(V+V_{B}+\phi_{B}\right)^{1 / 2}\right)}\right) d V$

where $\beta, \theta, \gamma$, and $\phi_{B}$ are the transistor current gain, mobility degradation factor, body factor, and surface potential and $V, V_{G}, V_{D}, V_{S}, V_{B}$, and $V_{F B}$ are the transistor channel, gate, drain, source and bulk voltages, and the flatband potential, respectively. Equation (1) can be rewritten as

$I_{D S}=\beta \int_{x_{2}}^{x_{1}}\left(\frac{\left(a_{o}+a_{1} x+a_{2} x^{2}\right) x}{b_{o}+b_{1} x+b_{2} x^{2}}\right) d x$

where

$a_{o}=2\left(V_{G}-V_{T o}+\gamma \sqrt{2 \phi_{B}}-V_{B S}+2 \phi_{B}\right)$

$a_{1}=-2 \gamma$

$a_{2}=-2$

$b_{o}=1+\theta\left(V_{G}-V_{T o}+\gamma \sqrt{2 \phi_{B}}-V_{B S}+2 \phi_{B}\right)$

$b_{1}=\theta \gamma$

$b_{2}=-\theta$

$V_{T o}=V_{F B}+\phi_{B}+\gamma \sqrt{2 \phi_{B}}$

$V_{B S}=\phi_{B}-V_{B}$

$x=\left(V-V_{B S}+2 \phi_{B}\right)^{1 / 2}$

$x_{1}=\left(V_{D}-V_{B S}+2 \phi_{B}\right)^{1 / 2}$

and

$x_{2}=\left(V_{S}-V_{B S}+2 \phi_{B}\right)^{1 / 2}$ 
By standard integral tables [10], (2) yields

$$
\begin{aligned}
& I_{D S}=\beta\left(d_{o}\left(x_{1}^{2}-x_{2}^{2}\right)+d_{1}\left(x_{1}-x_{2}\right)+d_{2} \ln \left(\frac{h_{1} x_{1}^{2}+h_{2} x_{1}+1}{h_{1} x_{2}^{2}+h_{2} x_{2}+1}\right)\right. \\
& \left.+h_{3} \ln \left(\frac{\left(h_{4} x_{1}+1\right)\left(h_{5} x_{2}+1\right)}{\left(h_{5} x_{1}+1\right)\left(h_{4} x_{2}+1\right)}\right)\right)
\end{aligned}
$$

where

$$
\begin{aligned}
& d_{o}=\frac{a_{2}}{2 b_{2}} \\
& d_{1}=\frac{a_{1} b_{2}-a_{2} b_{1}}{b_{2}^{2}} \\
& d_{2}=\frac{a_{o}}{2 b_{2}}-\frac{a_{1} b_{1}}{2 b_{2}^{2}}+a_{2}\left(\frac{b_{1}^{2}-b_{2} b_{o}}{2 b_{2}^{3}}\right) \\
& d_{3}=-\frac{a_{o} b_{1}}{2 b_{2}}+\frac{a_{1}\left(b_{1}^{2}-2 b_{2} b_{o}\right)}{2 b_{2}^{2}}+\frac{a_{2}\left(3 b_{o} b_{1} b_{2}-b_{1}^{3}\right)}{2 b_{2}^{3}} \\
& h_{1}=\frac{b_{2}}{b_{o}} \\
& h_{2}=\frac{b_{1}}{b_{o}} \\
& h_{3}=\frac{d_{3}}{\sqrt{b_{1}^{2}-4 b_{o} b_{2}}} \\
& h_{5}=\frac{2 b_{2}}{b_{1}+\sqrt{b_{1}^{2}-4 b_{o} b_{2}}} \\
& b_{1}-\sqrt{b_{1}^{2}-4 b_{o} b_{2}} \\
& h_{1} \\
& h_{1}
\end{aligned}
$$

and

$b_{1}^{2}>4 b_{o} b_{2}$

Table I shows calculated values for $d$ 's and $h$ 's for 
Values of the $d$ 's and $h$ 's.

\begin{tabular}{lrcc}
\hline parameter & value & parameter & value \\
\hline$d_{0}$ & 8.3333 & $h_{1}$ & -0.054158 \\
$d_{1}$ & 28.3333 & $h_{2}$ & 0.046034 \\
$d_{2}$ & 81.4861 & $h_{3}$ & -68.5993 \\
$d_{3}$ & -71.0908 & $h_{4}$ & 0.256871 \\
& & $h_{5}$ & -0.210837 \\
\hline
\end{tabular}

$V_{T o}=1.28 V, V_{G}=5 V, V_{S}=0, V_{B S}=-5 V, 2 \phi_{B}=0.7 V, \theta=0.12 V^{-1}, \gamma=0.85 V^{1 / 2}$

Although (3) is exact, it cannot yield closed-form expressions for the harmonic distortion components of the drain current, $I_{D S}$, resulting from a sinusoidal drain-to-source voltage $V_{D S}$. However, by using the following approximations

$\ln (1+x) \cong x-\frac{x}{2}+\frac{x^{3}}{3}-\frac{x^{4}}{4}+\frac{x^{5}}{5}$

and

$(1+x)^{1 / 2} \cong 1+0.5 x-0.125 x^{2}$

it is easy, though tedious, to express the drain current as

$I_{D S}=\beta\left(\alpha_{1} V_{D S}+\alpha_{2} V_{D S}^{2}+\alpha_{3} V_{D S}^{3}+\alpha_{4} V_{D S}^{4}\right)$

where

$$
\begin{aligned}
& \alpha_{1}=T_{1}+0.5 T_{2} \psi^{-1 / 2}+2 T_{3} \psi+1.5 T_{4} \psi^{1 / 2}+2.5 T_{5} \psi^{3 / 2} \\
& \alpha_{2}=-0.125 T_{2} \psi^{-3 / 2}+T_{3}+0.375 T_{4} \psi^{-1 / 2}+1.875 T_{5} \psi^{1 / 2} \\
& \alpha_{3}=-0.125 T_{4} \psi^{-3 / 2}-1.5 T_{5} \psi^{-1 / 2} \\
& \alpha_{4}=-0.125 T_{5} \psi^{-3 / 2} \\
& \psi=\sqrt{-V_{B S}+2 \phi_{B}}
\end{aligned}
$$

and

$$
\begin{aligned}
& T_{1}=d_{o}+d_{2} h_{1}-\frac{1}{2} h_{2}^{2} d_{2}-\frac{1}{2} h_{3} h_{4}^{2}+\frac{1}{2} h_{3} h_{5}^{2} \\
& T_{2}=d_{1}+d_{2} h_{2}+h_{3} h_{4}-h_{3} h_{5}
\end{aligned}
$$


$T_{3}--\frac{1}{2} d_{2} h_{1}^{2}-\frac{1}{4} h_{3}\left(h_{4}^{4}-h_{5}^{4}\right)$

$T_{4}=-d_{2} h_{1} h_{2}+\frac{1}{3} h_{3}\left(h_{4}^{3}-h_{5}^{3}\right)$

$T_{5}=\frac{1}{5} h_{3}\left(h_{4}^{5}-h_{5}^{5}\right)$

Table II shows calculated values of T's and $\alpha$ 's using the values of the parameters in Table I.

Using (4) the drain current $I_{D S}$ resulting from a drain-to-source voltage

$V_{D S}=V_{p} \sin \omega t$

can be expressed as

$I_{D S}=\beta\left(K_{o}+K_{1} \sin \omega t+K_{2} \cos 2 \omega t+K_{3} \sin 3 \omega t+K_{4} \cos 4 \omega t\right)$

where

$K_{o}=\frac{1}{2} \alpha_{2} V_{p}^{2}+\frac{3}{8} \alpha_{4} V_{p}^{4}$

$K_{1}=\alpha_{1} V_{p}+\frac{3}{4} \alpha_{3} V_{p}^{3}$

$K_{2}=-\frac{1}{2} \alpha_{2} V_{p}^{2}-\frac{1}{2} \alpha_{4} V_{p}^{4}$

$K_{3}=-\frac{1}{4} \alpha_{3} V_{p}^{3}$

and

TABLE II

Values of the $T$ 's and $\alpha$ 's.

\begin{tabular}{lccc}
\hline parameter & value & parameter & value \\
\hline$T_{1}$ & 4.57236 & $\alpha_{1}$ & 1.530526 \\
$T_{2}$ & $0.1526 \mathrm{E}-04$ & $\alpha_{2}$ & -0.235625 \\
$T_{3}$ & -0.078725 & $\alpha_{3}$ & 0.0168936 \\
$T_{4}$ & -0.398719 & $\alpha_{4}$ & $0.19344 \mathrm{E}-03$ \\
$T_{5}$ & -0.0210595 & & \\
\hline
\end{tabular}


$K_{4}=\frac{1}{8} \alpha_{4} V_{p}^{4}$

Using (8-11) the second, third, and fourth harmonic distortions can be expressed as

$H D_{2}=\frac{K_{2}}{K_{1}}$

$H D_{3}=\frac{K_{3}}{K_{1}}$

and

$H D_{4}=\frac{K_{4}}{K_{1}}$

\section{RESULTS}

Using (12)-(14) the harmonic performance of the fully-differential MOS voltagecontrolled resistor circuit shown in Fig. 1 [11] can be calculated. However, because of the fully-differential nature of the circuit, the second- and fourth-harmonic components will cancel and only the third-harmonic distortion will dominate. The results obtained, using the parameters of Tables I and II are shown in Fig. 2. From Fig. 2, it is obvious that for input voltages between $0.2 \mathrm{~V}$ and $4 \mathrm{~V}$ peak-to-peak, the third-harmonic distortion levels relative to the fundamental varies from $-91 \mathrm{~dB}$ to $-40 \mathrm{~dB}$. These trends are similar to the previously reported results $[5,11]$.

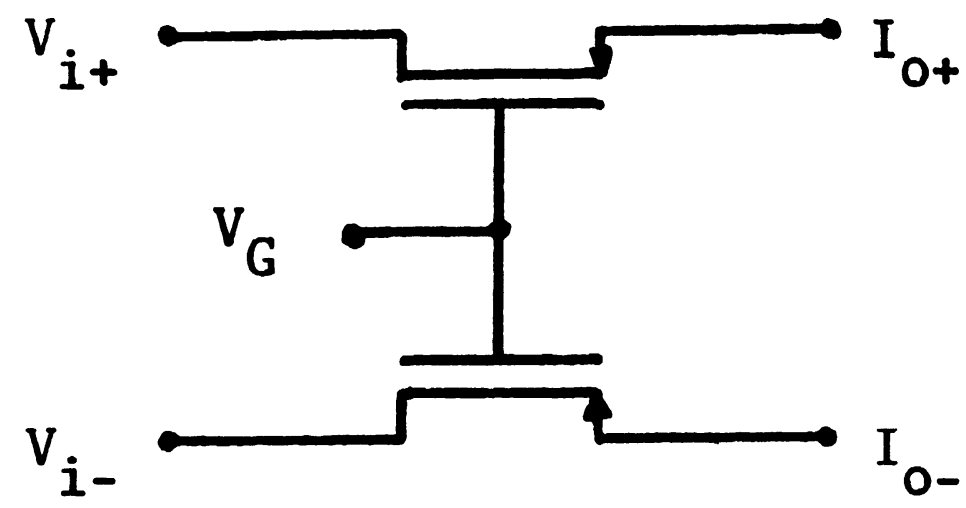

FIGURE 1 MOS Voltage-Controlled Resistor Circuit [11]. 


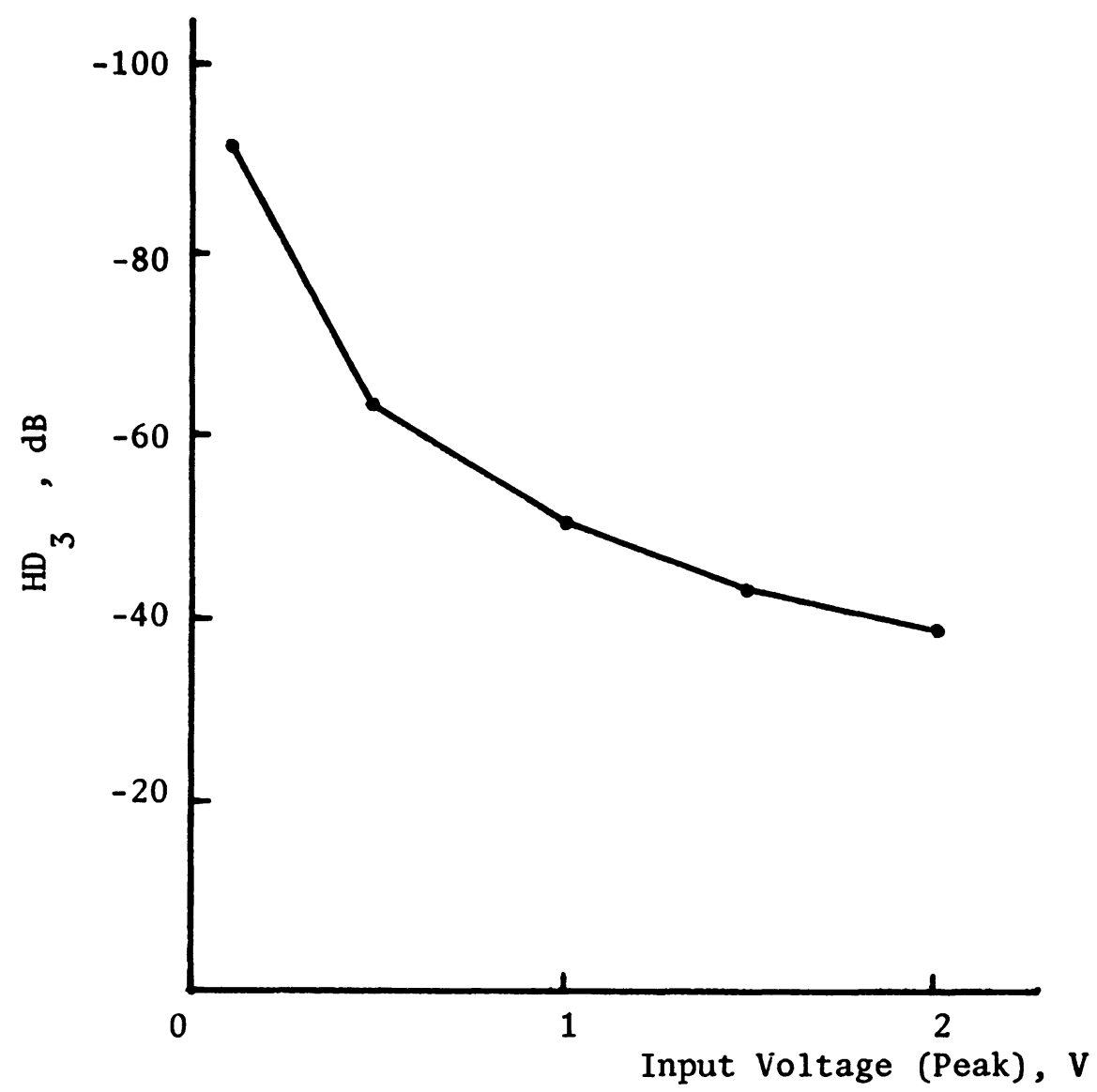

FIGURE 2 Third Harmonic Distortion for the Circuit of Fig. 1.

\section{CONCLUSION}

In this paper, a simple fourth-order polynomial expression has been presented for the dependence of the drain current of a MOS transistor on the drain-to-source voltage and other physical and dimensional parameters. Using this expression, closed-form expressions are obtained for predicting the harmonic performance of a MOS transistor operating in the triode region.

The closed-form expressions obtained in this paper can be used for quantitative analysis of the effect of different MOS transistor parameters on the harmonic distortion performance of MOS voltage-controlled-resistors and for selecting the values of the transistor parameters that will yield a prespecified distortion performance.

The results obtained in this paper are in fairly good agreement with previously published simulation results obtained using a modified version of SPICE [5]. 


\section{REFERENCES}

1. M. Banu and Y. Tsividis, Fully integrated active RC filters in MOS technology, IEEE Journal of Solid-State Circuits, Vol. SC-18, 1983, pp. 644-651

2. J.L. Pennock, CMOS triode transconductor for continuous-time active integrated filters, Electronics Letters, Vol. 21, 1985, pp. 817-818

3. P.J. Ryan and D.G. Haigh, Novel fully differential MOS transconductor for integrated continuoustime filters, Electronics Letters, Vol. 23, 1987, pp. 742-743

4. G. Merckel, J. Borel and N.Z. Cupcea, An accurate large-signal MOS transistor model for use in computer-aided design, IEEE Transactions on Electron Devices, Vol. ED-19, 1972, pp. 681-690

5. P.J. Ryan, Harmonic distortion in continuous-time filters, Electronics Letters, Vol. 25, 1989, pp. 1536-1537

6. M.H. White, F. Van de Wiele and J.-P. Lambot, High-accuracy MOS models for computer aided design, IEEE Transactions on Electron Devices, Vol. ED-27, 1980, pp. 899-906

7. G. Wilson and P.K. Chan, Analysis of nonlinearities in MOS floating resistor networks, IEE Proceedings-Circuits Devices Systems, Vol. 141, 1994, pp. 82-88

8. F.S. Shoucair and W.R. Patterson, Analysis and modeling of nonlinearities in VLSIMOSFET's including substrate effects, IEEE Transactions on Electron Devices, Vol. 40, 1993, pp. 1760-1767

9. M.T. Abuelma'atti, An improved analysis for the nonlinear performance of body-driven analog MOSFET circuits, Analog Integrated Circuits and Signal Processing, Vol. 5, 1994, pp. 175-181

10. G.A. Korn and T.M. Korn, Mathematical Handbook for Scientists and Engineers, McGraw-Hill Inc., New York, 1968 [Integrals 43-45, p. 932].

11. P.J. Ryan and D.G. Haigh, Novel fully differential MOS transconductor for integrated continuoustime filters, Electronics Letters, Vol. 23, 1987, pp. 742-743 

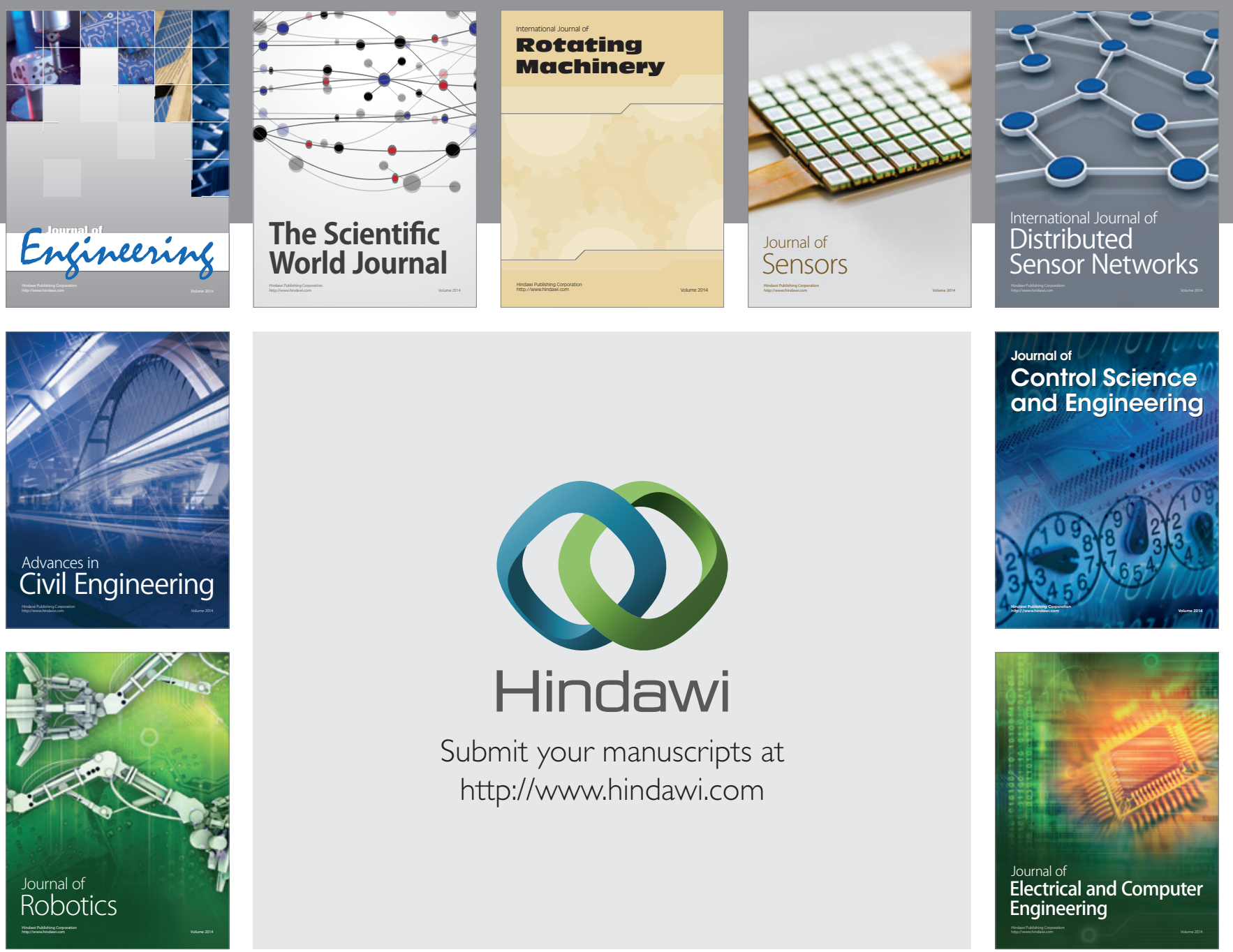

Submit your manuscripts at

http://www.hindawi.com
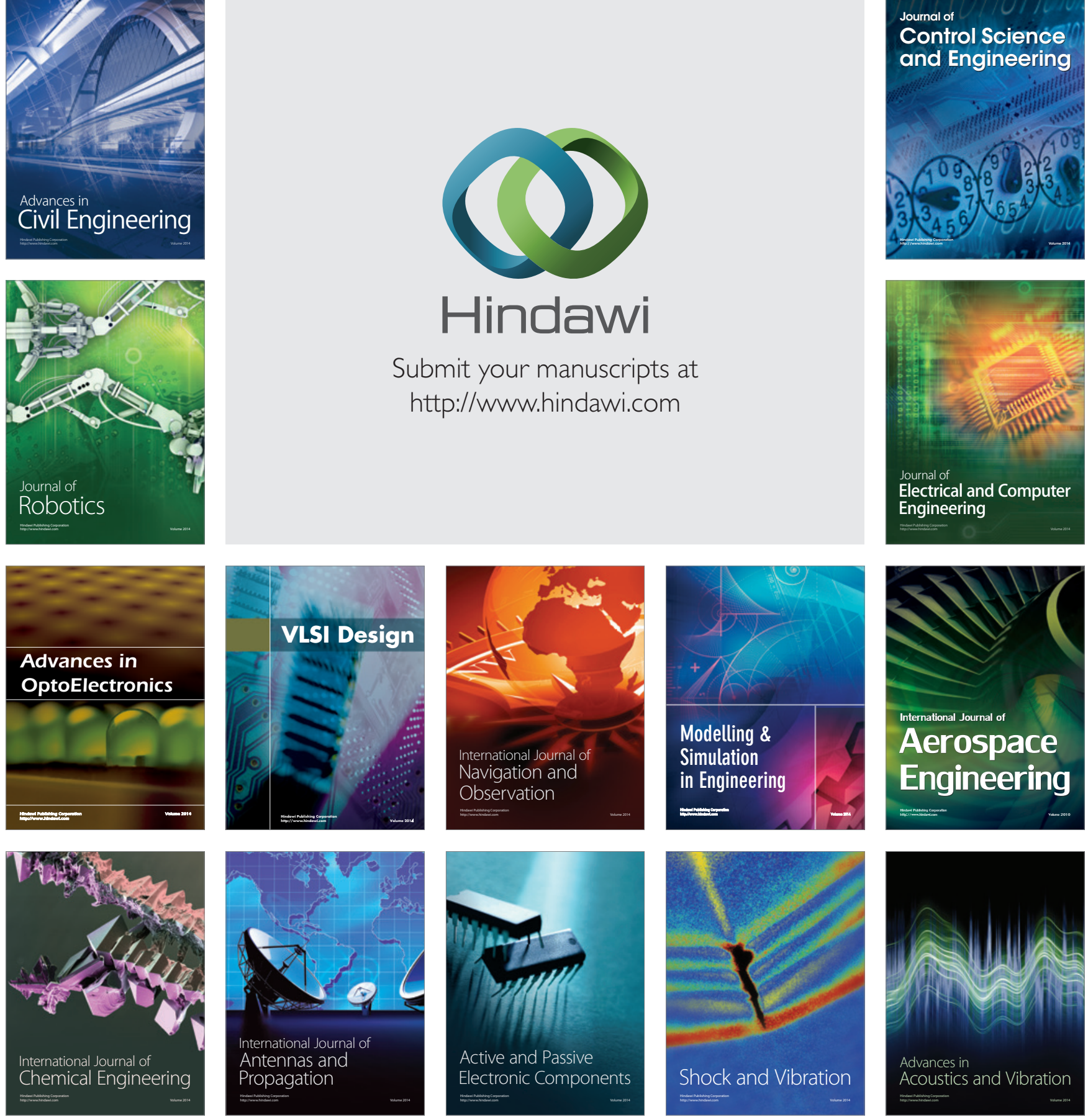